International Journal of Pavement Engineering

\title{
Environmental effects on the rheological properties of fine warm RAP-foamed bitumen mixtures using SATS conditioning protocol
}

Diana B. Sánchez, Gordon Airey, Silvia Caro \& James Grenfell

To cite this article: Diana B. Sánchez, Gordon Airey, Silvia Caro \& James Grenfell (2019):

Environmental effects on the rheological properties of fine warm RAP-foamed bitumen mixtures using SATS conditioning protocol, International Journal of Pavement Engineering, DOI: 10.1080/10298436.2019.1679365

To link to this article: https://doi.org/10.1080/10298436.2019.1679365

曲 Published online: 25 Oct 2019.

Submit your article to this journal $₫$

Q View related articles $[7$

Q View Crossmark data $\complement$ 


\title{
Environmental effects on the rheological properties of fine warm RAP- foamed bitumen mixtures using SATS conditioning protocol
}

\author{
Diana B. Sánchez ${ }^{* 1}$, Gordon Airey ${ }^{1}$, Silvia Caro ${ }^{2}$, and James Grenfell ${ }^{3}$ \\ 1: Nottingham Transportation Engineering Centre (NTEC), University of Nottingham. Nottingham, UK. \\ 2: Department of Civil and Environmental Engineering, Universidad de los Andes. Bogotá, Colombia. \\ 3: Australian Road Research Board (ARRB). Vermont, Victoria, Australia. \\ *bo-sanch@uniandes.edu.co
}

This paper uses the Saturation Ageing Tensile Stiffness (SATS) conditioning to evaluate the combined effects of moisture and oxidative ageing on the rheological characteristics of the Fine Aggregate Matrix (FAM) portion of various Foamed Bitumen Mixtures (FBMs) in combination with Reclaimed Asphalt Pavement (RAP) material. These FAM materials were produced at mixing temperatures of 90,120 and $160^{\circ} \mathrm{C}$ (i.e. half-warm, warm and hot processes, respectively). A fine Hot Mix Asphalt (HMA) with RAP - no foamed bitumen - was also manufactured for control purposes. The rheological properties of these fine mixtures were determined through Dynamic Mechanical Analysis (DMA) tests on specimens tested in their unconditioned and after SATS conditioning states. Rheology of the recovered binders of the four FAM mixes before and after SATS conditioning was also studied, and rheological parameters were used to link their long-term performance in terms of cracking susceptibility. The results showed an overall increase in the stiffness of the fine mixtures after the conditioning process, mainly due to oxidation of the bitumen. Although the half-warm and warm fine RAP-FBMs exhibited the highest increase in their complex shear modulus after SATS conditioning, rheological analysis of their corresponding binders indicated that their long-term performance in terms of cracking susceptibility might be superior to those of the hot RAP-FBM, and the HMA-RAP fine mixtures.

Keywords: Moisture, ageing, foamed bitumen mixtures, reclaimed asphalt pavement (RAP), fine aggregate matrix, asphalt mortar, SATS.

Word count: 8984

\section{Introduction}

Foamed bitumen is a road construction technique where the bitumen is heated at high temperatures (i.e. usually between $140-160^{\circ} \mathrm{C}$ ) and then foamed by combining it with cold water during mixture production. In its foamed state, the bitumen presents an increased volume that is up to 20 times its original volume, and lower viscosity than the same bitumen in its liquid state (Csanyi, 1957, Jenkins et al, 2000). These two conditions allow coating of aggregates at lower temperatures than those typically used for Hot Mix 
Asphalt (HMA) production with good workability conditions (Mallick et al., 2004). In addition, since only the bitumen is heated at high temperatures, this technique provides significant energy savings compared to traditional HMA (Bowering and Martin, 1976, Jenkins et al., 1999). This process is generally used for stabilisation of various types of materials, which include Reclaimed Asphalt Pavement (RAP) (i.e. cold recycling), and it can be accomplished in a central plant or can be applied on-site.

RAP materials are distressed asphalt pavements that have reached the end of their service lives, and are reclaimed to be used as part of the construction of new pavement structures, or rehabilitation of existing ones. The incorporation of this material in HMA reduces the amount of virgin bitumen and aggregates added to the final mixtures and, therefore, contributes to the conservation of natural and non-renewable resources (Copeland, 2011).

With the increasing experience in the use of FBMs and RAP materials in road infrastructure projects, it is natural for pavement engineers to combine these two technologies moving towards more sustainable road construction practices. In this sense, the advantages of using foamed bitumen technology along with RAP material include: 1) aid in compaction and workability of the recycled mixtures due to the viscosity reduction of the foamed bitumen, and 2) reduced RAP binder ageing due to lower production temperatures (D'Angelo et al. 2007). However, as any bituminous material used as part of surface courses, these mixtures are exposed in the field to the presence of moisture and oxidative ageing, which can result in loss of the durability of flexible pavements.

A number of studies in the literature have investigated the moisture susceptibility or oxidative ageing of asphalt mixtures containing high RAP content (e.g. Karlsson \& Isacsson 2006; Mogawer et al. 2012; Tran et al. 2012, Tarbox et al. 2012, Yin et al. 2017) and in foamed bitumen mixtures (FBMs) produced by direct water injection (e.g. Kim et al. 2012, Oyebisi, S 2014, Newcomb et al. 2015, Abbas et al, 2016, Kavussi et al. 2017, Raab et al, 2017). However, only a few research efforts have investigated the effects of these phenomena on recycled mixtures with foamed bitumen. In terms of moisture susceptibility, some of these efforts include the work by Shu et al. (2012) and Zhao et al. (2012) who found that with the incorporation of high RAP content, foamed bituminous mixtures exhibited satisfactory moisture resistance compared to a control mixture containing RAP with no foamed binder. In these studies, the authors attributed the beneficial effect of RAP on moisture resistance of the asphalt mixtures to stronger bonding of aged bitumen and aggregate particles, compared to that of the virgin bitumen 
and aggregate particles system, which renders the whole mixture to be less susceptible to moisture damage. In the study by Kusam et al. (2016), the authors evaluated the moisture susceptibility of FBMs with RAP content up to $40 \%$ and found that at $40 \%$ RAP, the Tensile Strength Ratio (TSR) (i.e. the retained tensile strength of the mixture after a moisture conditioning process) was below the passing criterion of $85 \%$. Thus, the authors recommended adding antistripping additives to enhance the moisture sensitivity of the final mixtures.

In another study, Palha et al. (2015) evaluated the water susceptibility of foamed mixtures containing $50 \%$ RAP produced at 134,138 and $160^{\circ} \mathrm{C}$, and a control mixture containing 50\%RAP with no foamed bitumen, produced at $160^{\circ} \mathrm{C}$. In this study, only the coarse fraction of the RAP material was heated together with the virgin aggregates to achieve the different mixing temperatures, while the fine RAP fraction was introduced at ambient temperature to mitigate further ageing of the RAP bitumen. In all cases, bitumen was foamed at $160^{\circ} \mathrm{C}$. The results showed that all recycled mixtures with foamed bitumen exhibited better moisture susceptibility resistance compared to the control mixture, which was attributed to improved workability of the former mixtures.

In terms of ageing, only the work by Perez-Martinez et al. (2017) was found to study the mechanical properties of a FBM containing 50\%RAP and those of a HMA (i.e. no foaming technique) containing 50\%RAP after being subjected to a laboratory ageing procedure. The authors monitored the change in the complex modulus of the mixtures with ageing and, among other results, found that the overall ageing evolution of both types of mixtures was similar.

As observed, the effects of ageing and moisture in mixtures with FBM and RAP is an area that requires further study since the results from previous works are still inconclusive. In addition, the combined impact of ageing and moisture in RAP-FBMs, which is relevant because it represents more realistic conditions of the processes that occur in the asphalt mixtures during their service life, has not been studied yet.

\section{Objective and methodology}

The objective of this study is to evaluate the combined effects of water and oxidative ageing on the linear viscoelastic properties of the Fine Aggregate Matrix (FAM) portion existing within various FBMs that incorporate $50 \%$ fine RAP material. The FAM mixtures were produced by direct water injection and manufactured at different production temperatures. Although the use of foamed bitumen by direct water injection 
with RAP material has been generally regarded as cold processes (i.e. foamed bitumen is sprayed onto the RAP material which is in a cold moist form), in this work hot foamed bitumen was combined with fine virgin aggregates and fine RAP material that were preheated at different temperatures to achieve final mixing temperatures around 90, 120 and $160^{\circ} \mathrm{C}$, since it has been demonstrated that temperature is a key variable influencing the mechanical response of the RAP-FBMs (Sánchez et al, 2019). A control fine HMA mixture with $50 \%$ fine RAP - no foamed bitumen - was also manufactured for comparison purposes.

FAM materials, also known as asphalt mortars, are herein defined as the blend of fine aggregates (particles below $1 \mathrm{~mm}$ ), mineral filler and bitumen. The maximum aggregate size of these materials was selected after reviewing existing literature, in which this value ranges between 1.18 and $2.36 \mathrm{~mm}$ (Masad et al., 2006, Caro et al., 2008, Valenta et al., 2010, Underwood \& Kim, 2013, Klug et al., 2018), as well as the sieve sizes currently used in the UK. The FAM material is particularly relevant in the mechanical response and performance of the full asphalt mixtures since it corresponds to the mortar that provides the bond between the coarse aggregates. However, since aggregates are hydrophilic materials - meaning that their affinity for water is greater than for bitumen - the presence of moisture tends to modify the properties of this matrix and, ultimately, to weaken their bonds with the coarse aggregates, accelerating the deterioration of the asphalt mixture (Caro et al., 2008, Tong et al., 2015, Vasconcelos et al., 2011). Furthermore, FAM materials contain high amounts of bitumen, which is mainly responsible for the stiffening of the asphalt mixtures during oxidation. Thus, the study of the environmental-related effects on these fine materials provide useful information about the durability of the corresponding full asphalt mixtures.

Although, FAM studies do not replace those conducted on full mixtures, this approach has gained popularity within the last fifteen years as it has been recognised that some deterioration processes, initiate and propagate within this fine matrix (Montepara et al. 2011). Thus, it is believed that tests conducted on FAM materials provide an initial indication of ways the phenomena would manifest in the corresponding asphalt mixture (Underwood \& Kim, 2013). Furthermore, the study at this scale is practical because given the homogeneity of the FAM material and the size of its components, it is possible to obtain the viscoelastic properties by means of testing small cylindrical samples with the use of a rheometer (a high precision equipment), at reduced costs, due to the significant 
decrease in the amount of time and materials required for the experiments. Details about the design, fabrication and testing of the FAM mixtures is presented in a later section.

The combined environmental conditioning of the fine mixtures was achieved by applying the Saturating Ageing Tensile Stiffness (SATS) protocol. SATS is the first experimental procedure developed to combine both ageing and water damage mechanisms in a single laboratory test protocol. The procedure has been successfully used to reproduce in the laboratory the loss of stiffness observed in asphalt mixtures in the field (Collop et al, 2004), and has been shown to correctly predict the susceptibility of bituminous mixtures to moisture damage processes, being able to distinguish between good and poor performing aggregate types (Collop et al., 2004, Airey et al., 2005, Grenfell et al, 2012).

The impact of the environmental effects was further studied on the linear viscoelastic properties of the recovered binders of all the FAM mixtures before and after being subjected to SATS conditioning. It is noteworthy to mention that it is the first time that the SATS conditioning protocol was used to evaluate the combined effects of moisture and laboratory ageing on FAM materials. Thus, the advanced experimental plan proposed in this work constitutes a novel and relevant contribution in the effort for assessing the influence of environmental-related effects on the mechanical response of fine RAP-FBMs.

\section{Design of FAM materials and characteristics of the fine RAP-FBMs}

The initial step in the design of the fine RAP-FBMs consisted of determining their gradation, which was derived from the gradation of the full asphalt mixture, a 0/14 mm size closed graded surface course based on the British Standard BS 4987-1:2005. Then, the FAM gradation was determined by normalising the fine particles to $1 \mathrm{~mm}$. Figure 1 shows the gradations of the full HMA mixture and of the corresponding FAM material. Limestone aggregates were used for all mixtures. Bitumen content for all the FAM mixtures was determined by means of a theoretical approach, which consisted of computing the volume of bitumen required to coat the fine particles using the volumetric properties of the full asphalt mixture (Masad et al, 2008). Based on this procedure, the bitumen content for FAM materials was $10.7 \%$ by mass of the total FAM mixture. 


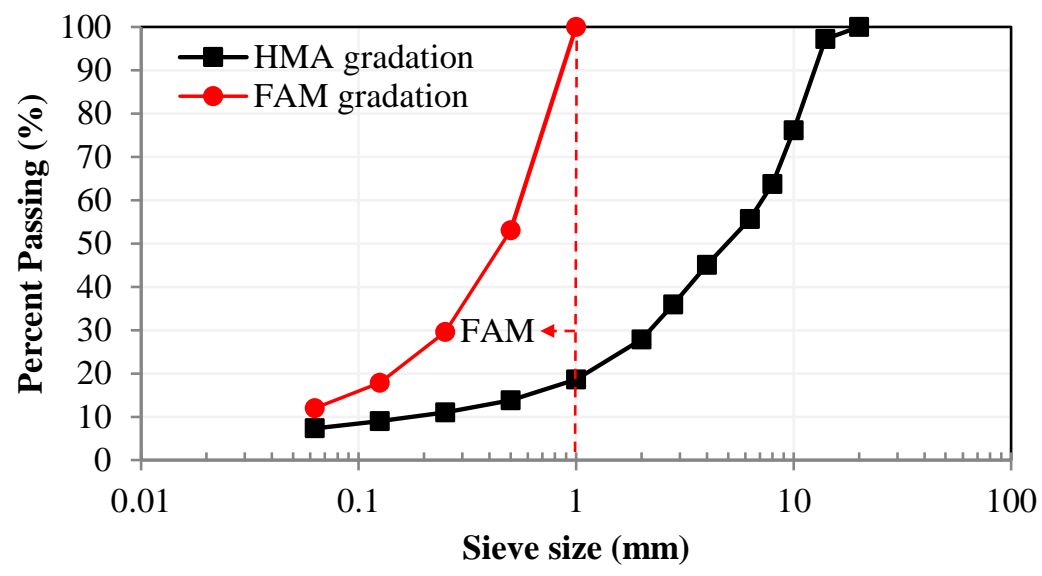

Figure 1 Gradation of the HMA and FAM mixtures

Initially, a FAM loose mixture with this gradation and bitumen content was produced using a 50/70 pen grade virgin bitumen. In order to characterise this bitumen, penetration at $25^{\circ} \mathrm{C}$ (BS 2000-49:2007), softening point (BS 2000-58:2007) and Fraass breaking point (BS 2000-80:2007) tests were performed. This FAM loose mixture was used to artificially produce the fine RAP material used for the preparation of the fine HMA-RAP and all the RAP-FBM fine mixtures. The procedure was based on previous studies conducted by Oke (2011), who conducted a comprehensive study that demonstrated the feasibility of this method to reproduce RAP material in the laboratory, and consisted of subjecting the loose mixture to heat in the oven for 32 hours at $105^{\circ} \mathrm{C}$. The advantage of using laboratory produced RAP material instead of actual RAP is that it allows the characteristics (i.e., aggregate gradation, bitumen content and its properties) of the material to be controlled. This reduces the variability commonly related with plant produced RAP material. The same tests to characterise the virgin bitumen were performed on the recovered bitumen from the fine RAP material. The properties of both binders are presented in Table 1.

Table 1 Properties of the virgin bitumen and recovered bitumen from RAP

\begin{tabular}{lccc}
\hline \multicolumn{1}{c}{ Bitumen } & $\begin{array}{c}\text { Penetration at } \mathbf{2 5}^{\mathbf{0}} \mathbf{C} \\
(\mathbf{d m m})\end{array}$ & $\begin{array}{c}\text { Softening Point } \\
\left({ }^{\mathbf{0}} \mathbf{C}\right)\end{array}$ & $\begin{array}{c}\text { Fraass breaking point } \\
\left({ }^{\mathbf{0}} \mathbf{C}\right)\end{array}$ \\
\hline Virgin bitumen & 66 & 48 & -8 \\
Recovered RAP bitumen & 31 & 59 & -8 \\
\hline
\end{tabular}

To account for the impact of the hard bitumen present in the fine RAP material, the fine mixtures were produced using a soft penetration grade bitumen, whose targeted properties were determined using the European blending laws (EN 13108-8:2005). For 
the purpose of this study, the properties of the required soft virgin bitumen were determined with the objective of obtaining an effective blended bitumen in the fine HMARAP and fine RAP-FBMs close to 50/70 pen. Table 2 shows the properties of each component of the final bitumen blend.

Table 2 Bitumen blend design for FAM mixtures with 50\% RAP content.

\begin{tabular}{lcc}
\hline \multicolumn{1}{c}{ Bitumen } & Penetration at $\mathbf{2 5}^{\mathbf{}} \mathbf{C}(\mathbf{d m m})$ & Softening Point $\left({ }^{\mathbf{}} \mathbf{C}\right)$ \\
\hline Virgin bitumen & 141 & 37 \\
Recovered bitumen* & 31 & 59 \\
Expected final bitumen blend & 66 & 48 \\
\hline *Recovered bitumen from the lab produced fine RAP material
\end{tabular}

Based on this blend design, a virgin bitumen of $133 \mathrm{dmm}$ penetration at $25^{\circ} \mathrm{C}$, softening point of $41^{\circ} \mathrm{C}$ - which are within the required values calculated in Table 2 -, and Fraass breaking point of $-14^{\circ} \mathrm{C}$, was used as the soft virgin bitumen in the fine HMARAP mixture, and was foamed to produce the fine RAP-FBMs.

The foamed bitumen used for the fabrication of the fine RAP-FBMs was produced using the Wirtgen WLB 10 laboratory foaming plant. In this procedure, foamed bitumen is generated by combining hot liquid bitumen with a controlled amount of cold water under high pressure in the expansion chamber of the foaming unit. Once foam is produced, it is sprayed directly from a special nozzle onto the fine RAP material and fine virgin aggregates. Foaming was performed by adding $3 \%$ water content to hot liquid bitumen heated at $160^{\circ} \mathrm{C}$.

Three fine FBMs containing 50\% fine RAP material (Foamed-RAP fine mixtures) using three different mixing temperatures of 90,120 and $160^{\circ} \mathrm{C}$ for half-warm, warm and hot applications respectively, were produced. In these mixtures, the virgin bitumen was heated at $160^{\circ} \mathrm{C}$ despite the variations in mixing temperatures, for foaming purposes. Thus, only the temperature of the fine RAP material and fine virgin aggregates was changed to achieve the final mixing temperature of the mixtures (i.e., 90,120 , and $160^{\circ} \mathrm{C}$ ), as shown in Table 3. It should be noted that the temperature of the constituent materials in the Foamed-RAP fine mixtures is higher than the target final mixing temperatures, to account for any heat loss during production of these fine mixtures, as specified in Table 3. A fine HMA-RAP control mixture (i.e., no foaming technology) with $50 \%$ fine RAP material, produced at $160^{\circ} \mathrm{C}$, was also manufactured.

In order to maintain the desired temperature of the fine aggregate particles for the three fine Foamed-RAP mixtures during mixing, a heated mixer was adapted to the 
foaming unit of the Wirtgen WLB 10, as suggested in the study conducted in the NCHRP Report 807 (Newcomb et al., 2015). For the fine HMA-RAP mixture, hand mixing was performed until the fine aggregates were fully coated. The amount of fine RAP material required for each mixture was heated up to the corresponding temperature (Table 3) for 6 hours prior to its incorporation with the virgin materials.

Table 3 Temperatures of the constituent materials for the FAM mixtures produced

\begin{tabular}{lcccc}
\hline \multicolumn{1}{c}{ Mixture type } & $\begin{array}{c}\text { Bitumen } \\
\mathbf{T}\left[{ }^{\circ} \mathbf{C}\right]\end{array}$ & $\begin{array}{c}\text { Fine virgin } \\
\text { aggregates } \mathbf{~}\left[{ }^{\circ} \mathbf{C}\right]\end{array}$ & $\begin{array}{c}\text { Fine RAP } \\
\mathbf{T}\left[{ }^{\circ} \mathbf{C}\right]\end{array}$ & $\begin{array}{c}\text { Mixing } \\
\mathbf{T}\left[{ }^{\circ} \mathbf{C}\right]\end{array}$ \\
\hline Fine HMA-RAP & 160 & 160 & 160 & 160 \\
Fine foamed-RAP 90C & 160 & 110 & 110 & 90 \\
Fine foamed-RAP 120C & 160 & 140 & 140 & 120 \\
Fine foamed-RAP 160C & 160 & 180 & 160 & 160 \\
\hline
\end{tabular}

\section{Experimental procedures}

This section describes the FAM mixtures' production, the SATS conditioning protocol adapted for FAM materials, and the testing procedures followed to determine the linear viscoelastic properties of the fine RAP-FBMs and their recovered binders.

\subsection{FAM specimen preparation}

Initially, one cylindrical specimen for each FAM mixture with dimensions of $100 \mathrm{~mm}$ in diameter by $60 \mathrm{~mm}$ in height was compacted in the laboratory using the Superpave gyratory compactor (SGC) following the BS EN 12697-31:2007 (Figure 2a), following the procedure proposed by Masad et al., 2016. For this study, the specimens were compacted targeting a density of $2056 \mathrm{~kg} / \mathrm{m}^{3}$, which corresponds to a target air void content of the compacted FAM specimens of 10\%; a typical value used in existing studies that focus on this material (Masad et al., 2008, Sánchez et al., 2017). It should be noted that all the fine mixtures reached the targeted values and were workable at the various production temperatures. Then, the top and bottom parts of the $100 \mathrm{~mm}$ diameter SGC FAM specimen were trimmed to obtain a specimen of $50 \mathrm{~mm}$ in height, and four cylindrical samples of $50 \mathrm{~mm}$ in height by $12.5 \mathrm{~mm}$ in diameter were extracted from the centre of the SGC core using a coring barrel (Figure $2 b$ ). These samples were retained for Dynamic Mechanical Analysis (DMA) testing for the 'initial' or 'control' condition (i.e. prior to environmental conditioning). 


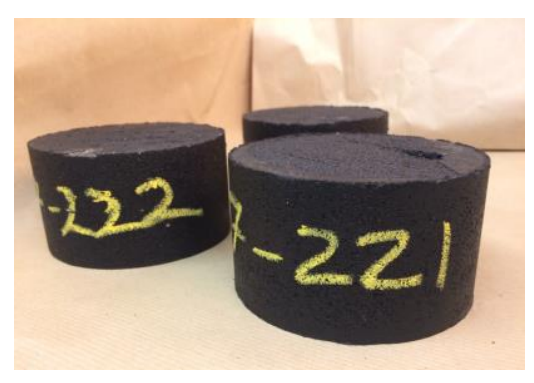

a)

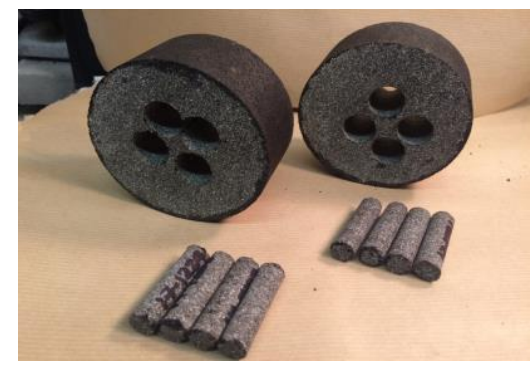

b)

Figure 2 FAM specimen preparation: a) SGC specimens, and b) cored cylindrical samples for mechanical testing for the initial condition

\subsection{SATS conditioning procedure}

The SATS test was developed at The University of Nottingham by Collop et al. (2004) and Airey et al. (2005), and is based on the principle of combining ageing with moisture conditioning by ageing compacted asphalt mixture specimens at an elevated temperature and pressure in the presence of moisture. A pressure vessel is used to hold five specimens of $100 \mathrm{~mm}$ in diameter by $60 \mathrm{~mm}$ in thickness in a custom-made container as shown in Figure 3. In this figure, $P_{1}, P_{2}$ etc. refer to the position of the specimens in the vessel.

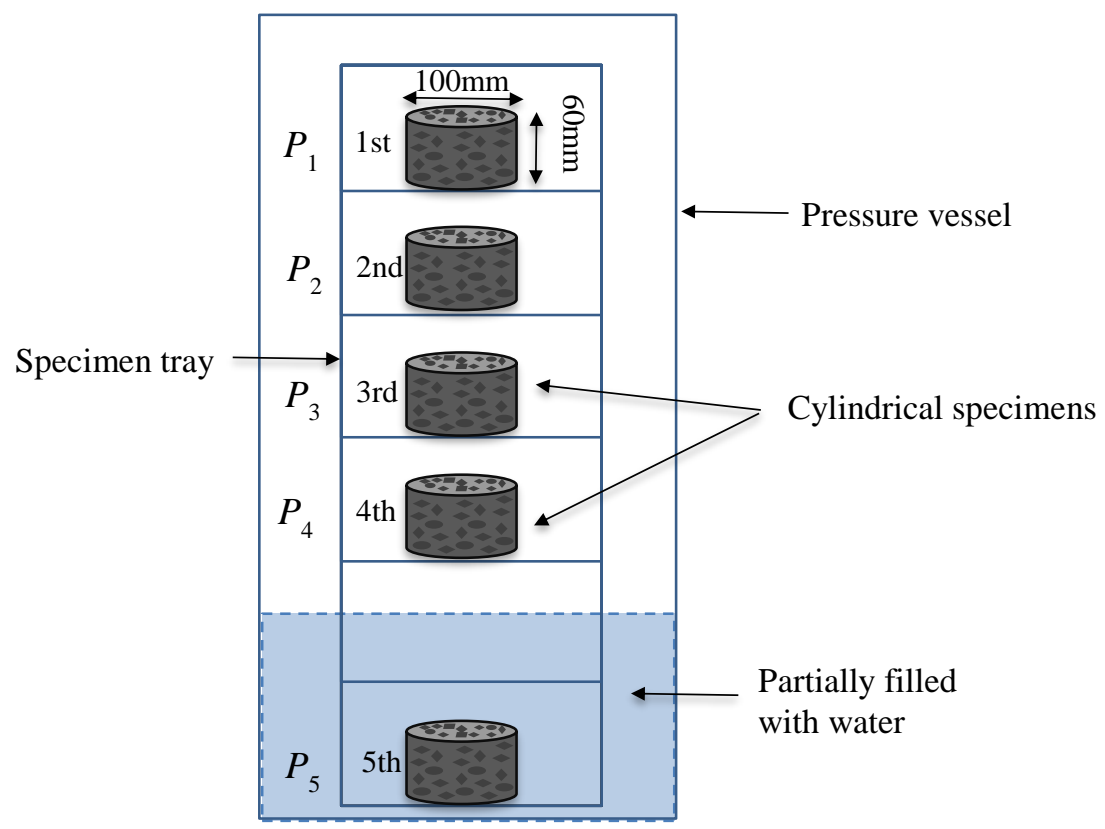

Figure 3 Schematic of the specimens in the SATS pressure vessel and specimen tray (modified after Airey et al. 2005)

During the test, the water in the pressure vessel condensates on the underside of the top lid causing 'dripping' onto the top specimen. There is then a cascading effect where smaller amounts of water progressively ‘drip' onto the specimens below, resulting 
in a decrease in the retained saturation level for specimens that are located lower down inside the pressure vessel (Grenfell et al., 2012). For the purpose of this study, the SATS procedure developed by Collop et al. (2004) and Airey et al. (2005) was adapted for FAM materials, as follows:

1. The dry mass of the cored SGC specimen is determined.

2. The cored specimen is then immersed in distilled water at $20^{\circ} \mathrm{C}$ and vacuum saturated using a residual pressure of $65 \mathrm{kPa}$ for $30 \mathrm{~min}$.

3. The wet mass of the specimen is determined, and the percentage saturation is calculated, referred to as 'initial saturation'. The specimen is then wrapped in a wire mesh to avoid slump during conditioning (Figure 4a).

4. The SATS pressure vessel is partially filled with distilled water until the level is between the fourth and fifth position ( $P_{4}$ and $P_{5}$ in Figure 3$)$. The pressure vessel and the water are maintained at the target temperature of $85^{\circ} \mathrm{C}$ for at least $2 \mathrm{~h}$ prior to introducing the specimens.

5. The saturated asphalt specimen is then placed into the pressure vessel, in the top position of the specimen tray ( $P_{1}$ in Figure 3$)$. Although the standard SATS test holds five cores at a time, as explained previously, for the purpose of this study, and since only one SGC specimen is manufactured per FAM mixture, the top position was selected to place the FAM specimen for each fine mixture (Figure $4 b$ ), as it is the most critical due to the moisture flux processes within the container. This means that no specimens were placed in the $P_{2}-P_{5}$ positions of the specimen tray. The vessel is then sealed and the air pressure is gradually raised to $2.1 \mathrm{MPa}$.

6. The specimen is maintained at these testing conditions (i.e. $2.1 \mathrm{MPa}$ and $85^{\circ} \mathrm{C}$ ) for 65 hours.

7. After $65 \mathrm{~h}$, the target vessel temperature is reduced to $30^{\circ} \mathrm{C}$ and the vessel is left to cool down for $24 \mathrm{~h}$. When the temperature has decreased to $30^{\circ} \mathrm{C}$ (after the $24 \mathrm{~h}$ cooling period), the air pressure is gradually released. When the vessel has achieved atmospheric pressure, it is opened and the specimen is removed. The specimen is then surface dried and weighed in air. The percentage saturation calculated at this stage is referred to as the 'retained saturation'.

Once the conditioning process was finished, four cylindrical samples of $50 \mathrm{~mm}$ in height by $12.5 \mathrm{~mm}$ in diameter were extracted from the outer part of the specimen avoiding contact with the already cored holes (Figure 4c), using a coring barrel. These samples were retained for DMA testing for the 'final' condition (i.e. environmental 
condition). The decision to extract the small cylindrical samples from the SCG core after SATS conditioning was to avoid potential disintegration of the small individual specimens if placed directly in the SATS during the conditioning process.

Thus, the conditioning procedure involves the same method to the standard testing conditions (Collop et al. 2004, Airey et al. 2005) except that only one FAM SGC specimen was conditioned at the $P_{1}$ position, and eight small cylindrical FAM samples were obtained afterwards for further DMA testing (i.e., four to be tested before conditioning and four after SATS conditioning), to obtain their linear viscoelastic properties.

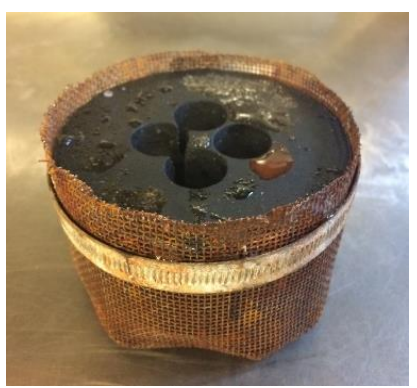

a)

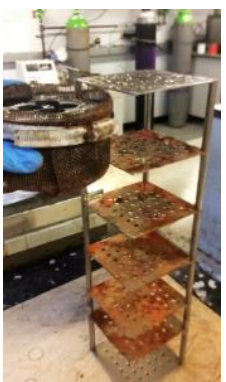

b)

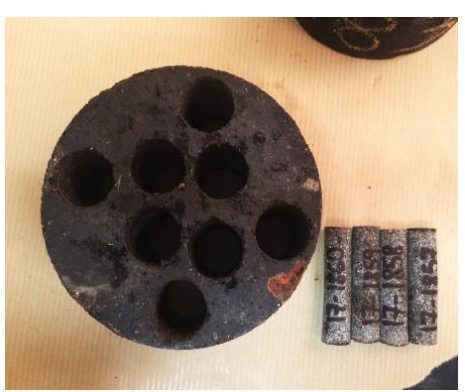

c)

Figure 4 SATS conditioning for FAM material: a) SGC - FAM specimen embedded in a metal mesh, b) specimen placed in the top position of the specimen tray, and c) cored FAM samples for testing after SATS conditioning.

\subsection{Test procedures to determine the linear viscoelastic properties of the fine RAP-}

\section{FBMs and their recovered binders}

As explained previously, the experimental plan on the environmental effects on the properties of the RAP-FBMs included two different efforts: 1) the quantification of the mechanical properties of the fine portion of the RAP-FBMs in the initial and final conditions, and 2) the determination of the rheological properties of their recovered binders after the SATS conditioning. While the first effort provides initial information of the material in terms of its competence to resist the environmental effects when used as part of the surface course in pavement structures, the latter complements this information by offering evidence of the changes that occurred in the bitumen due to the selected conditioning protocol in terms of its rheological properties. Thus, this section describes the test procedures to determine the linear viscoelastic properties of both materials.

For the FAM samples, their rheological properties were measured by means of the DMA testing technique, which has been widely used to characterise the linear and 
non-linear viscoelastic properties of these materials, as well as mechanical deterioration processes under cyclic loading (Masad et al., 2006, 2008; Caro et al., 2008, 2012; Caro, Sánchez, \& Caicedo, 2015; Gudipudi \& Underwood, 2015; Tong et al., 2013, 2015, Sánchez et al. 2017, Klug et al., 2018, Sánchez et al., 2019). The procedure consists of using a rheometer with a fixture configuration for solid materials, to apply oscillation loading to the small FAM cylindrical specimens (i.e. $50 \mathrm{~mm}$ in height by $12.5 \mathrm{~mm}$ in diameter). For the purposes of this study, frequency and temperature sweep tests were conducted using a constant strain amplitude of $6.5 \times 10^{-3} \%$, a value that has been proved to maintain the response of FAM materials within the linear viscoelastic range (Caro et al. 2008, Sánchez et al. 2017), using loading frequencies from $0.5-15.9 \mathrm{~Hz}$, and varying temperatures from $15-65^{\circ} \mathrm{C}$ with $10^{\circ} \mathrm{C}$ increments. Figure 5 shows the specimens' preparation, which includes gluing metallic holders to the cylindrical samples for further mounting in the rheometer, and the DMA testing set-up.

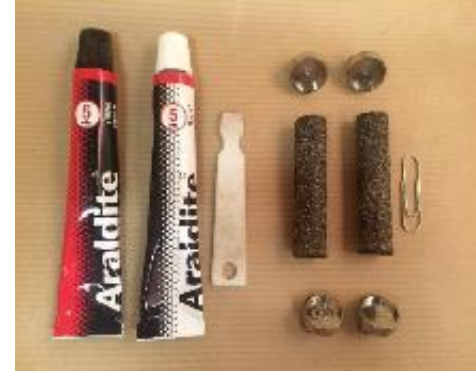

(a)

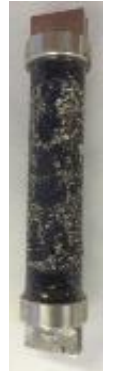

(b)

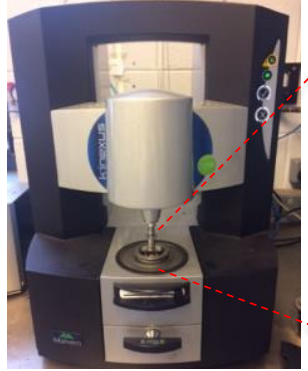

(c)

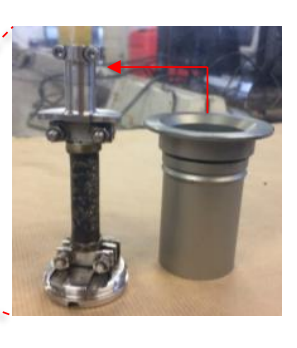

Figure 5 FAM Specimens' preparation and DMA testing set-up: a) gluing process, b) sample with metallic holders, and c) sample mounting in the rheometer.

For the binders recovered from all evaluated FAM mixtures before and after SATS conditioning, dynamic shear tests were conducted using the rheometer with the parallel plates configuration. The binders were tested in a strain control mode $(0.2 \%$ to $0.8 \%$, depending on temperature) at varying temperatures from 5 to $75^{\circ} \mathrm{C}$ at $5^{\circ} \mathrm{C}$ intervals, and loading frequencies between 0.1 and $10 \mathrm{~Hz}$. For temperatures between 5 to $45^{\circ} \mathrm{C}$, the parallel plate geometry of $8 \mathrm{~mm}$ and $2 \mathrm{~mm}$ gap was used. For temperatures between 25 to $75^{\circ} \mathrm{C}$, the parallel plate geometry of $25 \mathrm{~mm}$ and $1 \mathrm{~mm}$ gap was used. The data for each binder from 25 to $45^{\circ} \mathrm{C}$ were selected after plotting their Black diagrams (i.e. plots of phase angle, $\delta$ vs. complex shear modulus, $\left.\left|G^{*}\right|\right)$ following the approach described by Airey (2002). 


\section{Results on the fine RAP-FBMs}

\subsection{Air void content and saturation levels}

Table 4 presents the average air void content values of the eight small cored specimens for each mixture, which was determined from their volumetric properties. This table shows that although there are some differences in the air void content among the fine mixtures, the standard deviations were between $0.48 \%$ and $0.66 \%$, which are considered acceptable for the purpose of this study.

Table 4 Average air void results for the small cored specimens for all FAM mixtures

\begin{tabular}{lcc}
\hline \multicolumn{1}{c}{ FAM mixture type } & Mean air void content (\%) & Standard deviation (\%) \\
\hline HMA-RAP & 9.8 & 0.50 \\
Foamed-RAP 90C & 9.5 & 0.48 \\
Foamed-RAP 120C & 9.6 & 0.66 \\
Foamed-RAP 160C & 10.1 & 0.60 \\
\hline
\end{tabular}

On the other hand, Table 5 shows the saturation levels (before and after SATS conditioning) for all the fine mixtures, which were measured on the SGC cores for each mixture before coring the four small specimens for testing after SATS conditioning. Although these values do not necessarily represent the actual saturation levels within the individual small cylindrical testing specimens, they were measured to obtain reference values. Table 5 shows that there is high variability in the retained saturation among the fine mixtures (approximately 28 to $46 \%$ ). Although all fine mixtures followed the same mixture design, these results imply that there might be differences in the internal composition and structure of each fine mixture due to different production temperatures, and this might have an influence in the results. Also, the average values of retained saturation obtained for these FAM materials are lower than those reported in the literature for full asphalt mixtures for this type of conditioning process. For example, Airey et al. (2005) reported a retained saturation level of $90 \%$ for a Dense Bitumen Macadam (DBM) base material specimen located in position $P_{1}$ of the specimen tray after SATS conditioning. Similarly, Airey et al. (2007) and Grenfell et al. (2015) reported retained saturation levels between 60 and $80 \%$ for DBM base material specimens located in the same position $\left(P_{1}\right)$ of the SATS specimen tray. These results indicate that the difference in the volumetric composition of the FAM materials compared to those of full asphalt mixtures, especially in terms of the larger amounts of bitumen and the potential existence 
of smaller pore sizes in the FAM mixtures, might impact the rheological results. The results also suggest the convenience of conducting future studies devoted at determining proper sets of SATS variables that could be used to achieve higher saturation levels in these fine materials.

Table 5 Saturation levels of the SGC specimens for all the FAM mixtures

\begin{tabular}{lcc}
\hline \multicolumn{1}{c}{ FAM Mixture } & $\begin{array}{c}\text { Initial saturation } \\
(\boldsymbol{\%})\end{array}$ & $\begin{array}{c}\text { Retained saturation } \\
(\boldsymbol{\%})\end{array}$ \\
\hline HMA-RAP & 53.5 & 46.0 \\
Foamed-RAP 90C & 30.4 & 28.2 \\
Foamed-RAP 120C & 45.6 & 43.1 \\
Foamed-RAP 160C & 38.2 & 36.1 \\
\hline
\end{tabular}

\subsection{Effects of SATS conditioning protocol on the linear viscoelastic properties of the fine mixtures}

Using information from the frequency and temperature test results, the average master curves of $\left|G^{*}\right|$ for each fine mixture were constructed. Figures 6a and b present, respectively, the $\left|G^{*}\right|$ master curves at a reference temperature of $25^{\circ} \mathrm{C}$ for the initial and final conditions (i.e. before and after SATS conditioning), constructed using the average values of the four tested specimens for each mixture at each condition.

a)

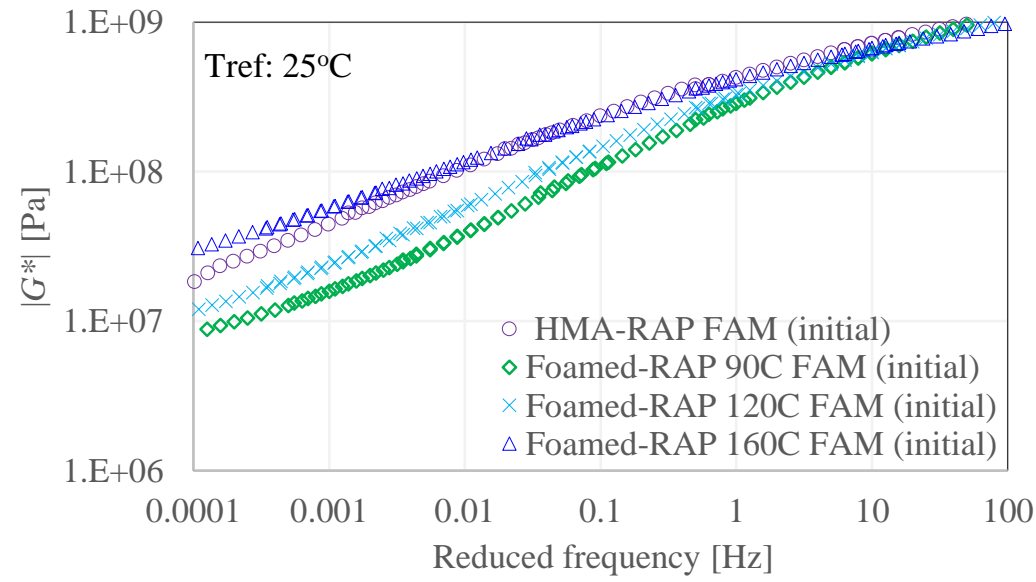


b)

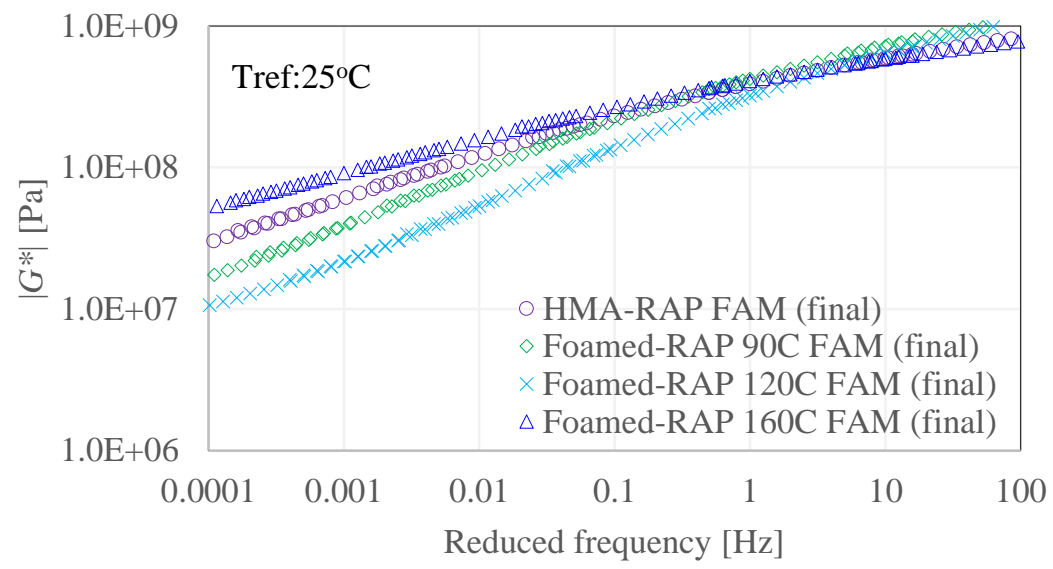

Figure 6 Master curves of $\left|G^{*}\right|$ for all fine RAP-FBMs for a) before, and b) after SATS conditioning.

At the initial condition, Figure 6a shows that all the fine mixtures approach the same stiffness at high frequencies, with larger differences at low frequencies. At low frequencies, the Foamed-RAP fine mixture manufactured at $160^{\circ} \mathrm{C}$ and the HMA-RAP fine mixture manufactured at the same temperature, exhibit the highest $|G *|$ values, followed by the Foamed-RAP fine mixture manufactured at $120^{\circ} \mathrm{C}$, and the Foamed-RAP fine mixture manufactured at $90^{\circ} \mathrm{C}$. For instance, at a frequency of $0.001 \mathrm{~Hz}$, their $\left|G^{*}\right|$ values are $5.8 \times 10^{7}, 4.5 \times 10^{7}, 2.1 \times 10^{7}$ and $1.6 \times 10^{7} \mathrm{~Pa}$, respectively. These results indicate that the stiffness of the Foamed-RAP fine mixtures increases with an increase in mixing and production temperature. Since all the fine mixtures followed the same mixture design, these results suggest that increasing the mixing temperature (i.e., subjecting the fine RAP material and fine virgin aggregates to higher temperatures prior to the combination with foamed bitumen - heated at $160^{\circ} \mathrm{C}$ in all cases -) might have led to higher contribution of the RAP bitumen in the total RAP-virgin bitumen blend, and also to further ageing of the RAP binder, as already found by Sánchez et al. (2019).

After the conditioning protocol, Figure $6 \mathrm{~b}$ shows that the fine mixtures produced at $160^{\circ} \mathrm{C}$ (i.e., Foamed-RAP and HMA-RAP fine mixtures) still exhibit the best mechanical capacity at low reduced frequencies. However, the $\left|G^{*}\right|$ curves of the FoamedRAP fine mixture manufactured at $90^{\circ} \mathrm{C}$, and the Foamed-RAP fine mixture manufactured at $120^{\circ} \mathrm{C}$ are interchanged. At high frequencies, the Foamed-RAP and HMA-RAP fine mixtures manufactured at $160^{\circ} \mathrm{C}$, exhibit lower $\left|G^{*}\right|$ values after SATS conditioning with respect to those of the Foamed-RAP fine mixtures manufactured at 90 and $120^{\circ} \mathrm{C}$. Nevertheless, the former fine mixtures showed a pronounced flattening effect 
on the $\left|G^{*}\right|$ master curves after SATS conditioning (Figure 6b), which results in lower $\left|G^{*}\right|$ values with respect to those of the latter fine mixtures, at these high frequencies.

In order to further analyse the differences in the environmental related effects of the fine RAP-FBMs, $\left|G^{*}\right|$ ratios were calculated by dividing the $\left|G^{*}\right|$ values obtained at the final condition (i.e., after SATS conditioning or $\left|G^{*}\right|_{\text {final }}$ ) to those of the initial condition $\left(\left|G^{*}\right|_{\text {initial }}\right)$ at all testing temperatures and at $10 \mathrm{~Hz}$, which is a typical frequency value used in pavement design. These results are presented in Figure 7. Higher $\left|G^{*}\right|$ ratios in this figure indicate a higher impact of the environmental-related effects on the dynamic modulus of the material (i.e., $\left|G^{*}\right|_{\text {final }}$ is larger in comparison to $\left|G^{*}\right|_{\text {initial }}$ ).

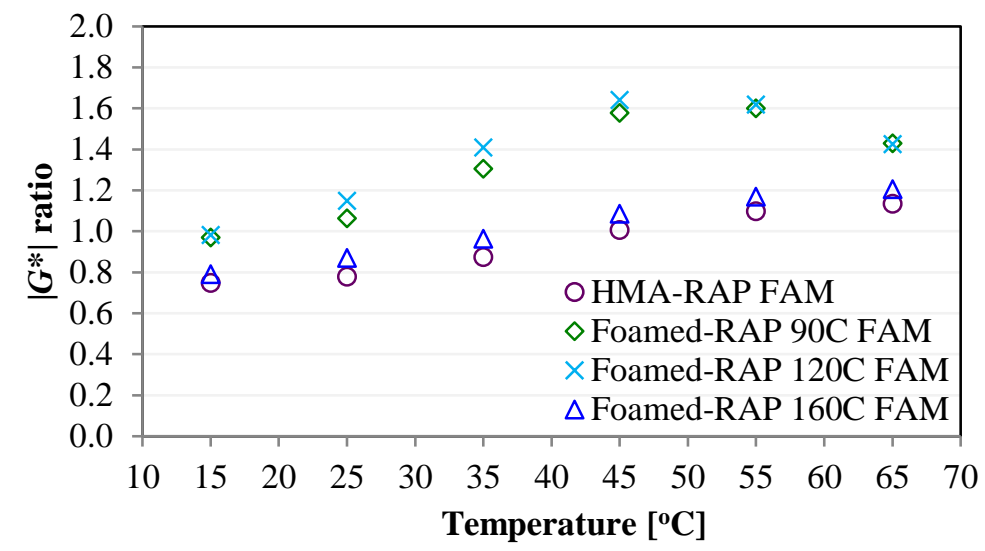

Figure $7\left|G^{*}\right|$ ratio $\left(\left|G^{*}\right|_{\text {initial } /}\left|G^{*}\right|_{\text {final }}\right)$ values at $10 \mathrm{~Hz}$ for all temperatures

This figure shows that the control HMA-RAP fine mixture consistently exhibited the lowest $\left|G^{*}\right|$ ratios at all temperatures, meaning that this fine mixture was the least affected by the environmental conditioning process. When the fine RAP mixtures are produced using the foaming technique, the $\left|G^{*}\right|$ ratios increase with respect to those of the reference HMA-RAP fine mixture, and this increase changes with production temperature. For instance, the $\left|G^{*}\right|$ ratios of the fine Foamed-RAP mixtures manufactured at 90 and $120^{\circ} \mathrm{C}$ exhibited an average increase (among all temperatures) of 41 and $46 \%$ with respect to the control fine HMA-RAP mixture, respectively, indicating low differences between these production temperatures but high susceptibility to the environmental related effects. It is also interesting to notice that for these fine mixtures, the $\left|G^{*}\right|$ ratios increase with temperature up to a maximum value at $45^{\circ} \mathrm{C}$, which corresponds to intermediate frequencies, after which they decrease and tend to become closer to those of the fine mixtures manufactured at $160^{\circ} \mathrm{C}$. This indicates that at high temperatures (low frequencies) the behaviour of all fine mixtures is comparable. 
Conversely, the $\left|G^{*}\right|$ ratio of the fine Foamed-RAP mixture manufactured at $160^{\circ} \mathrm{C}$ exhibited an average increase of only $8 \%$ with respect to that of the fine HMA-RAP.

These results show not only that a stiffening effect occurred in the materials after the SATS conditioning protocol, but that it occurred to a different extent in each fine mixture where the fine mixtures with a high initial stiffness exhibited less ageing than those of a lower initial stiffness.

As an aside and support for the observations above, previous physical, rheological and chemical tests conducted on a virgin bitumen subjected to a moisture Pressure Ageing Vessel (PAV) test, demonstrated that moisture condition together with heat, oxygen and pressure promotes ageing of the bitumen, resulting in a stiffer and more brittle binder (Ma et al., 2011). In another study, rheological and chemical tests conducted on a virgin bitumen submerged in distilled water for periods of up to nine months, demonstrated that the moisture conditioning process generated changes in the chemistry of the material that could be attributed to oxidative ageing (Figueroa-Infante, 2015). Results from other studies that evaluated the effect of ageing and moisture on the mechanical and resistance characteristics of asphalt mixtures found that ageing resulted in higher stiffness and lower toughness of the asphalt mixtures, while the effect of water was minimal, which the authors attributed to the water immersion conditions not been aggressive enough for the evaluated mixture (López-Montero and Miró, 2016). Furthermore, results from previous studies on the combined effects of ageing and moisture sensitivity on the stiffness of high modulus base materials, showed an initial stiffening effect of the mixtures, followed by a decrease in modulus after various combined ageing and moisture sensitivity regimes (Airey et al., 2005).

Therefore, it is speculated that the stiffening effects that were observed in this study for the fine mixtures are mainly attributed to oxidation effects in the bitumen. Furthermore, the fact that FAM materials are composed of a rich bitumen phase and fine particles, with smaller sized air voids compared to those of conventional HMA, could impact the rate at which moisture reaches the inner portion of the specimen. This moisture degrades the adhesive bonds between the bitumen and the fine aggregate particles, allowing damage to occur under the presence of moisture (i.e., reduction in the magnitude of the $\left|G^{*}\right|$ values), whereas the elevated temperatures would certainly lead to some ageing and stiffening of the materials, which generates the overall increase in $\left|G^{*}\right|$ in the master curves. This statement is also supported by the fact that the saturation levels of the SGC fine specimens are lower than those typically obtained for HMA mixtures, 
indicating that water may only have reached a limited portion of the small FAM testing specimens.

\section{Effects of SATS conditioning protocol on the recovered binders from the fine RAP-FBMs}

The average $\left|G^{*}\right|$ master curves for two replicates, at a reference temperature of $25^{\circ} \mathrm{C}$, obtained for the recovered binders of each fine mixture at the initial condition and after SATS conditioning are shown in Figures $8 \mathrm{a}$ and $\mathrm{b}$ respectively.

a)

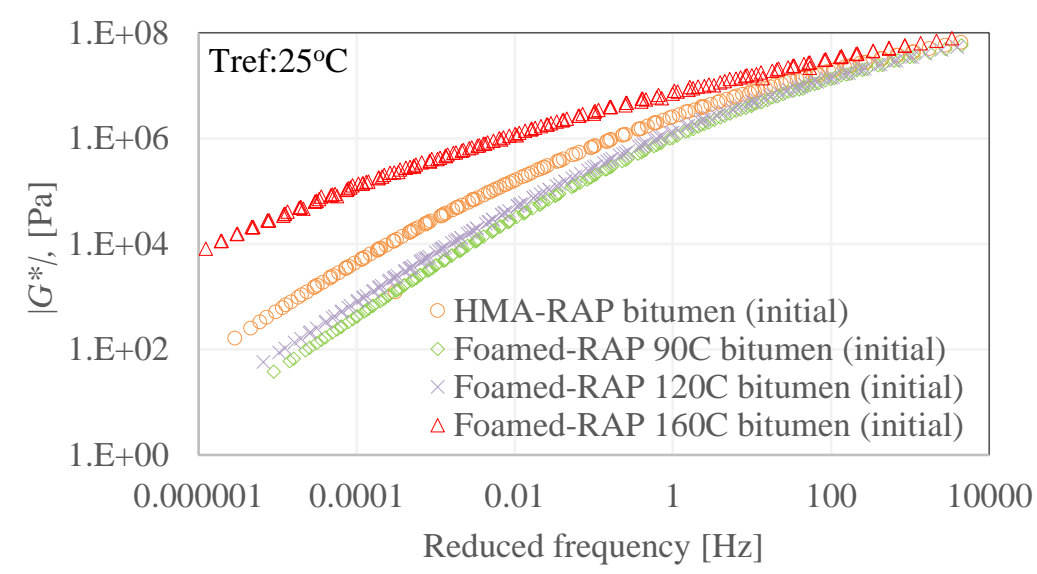

b)

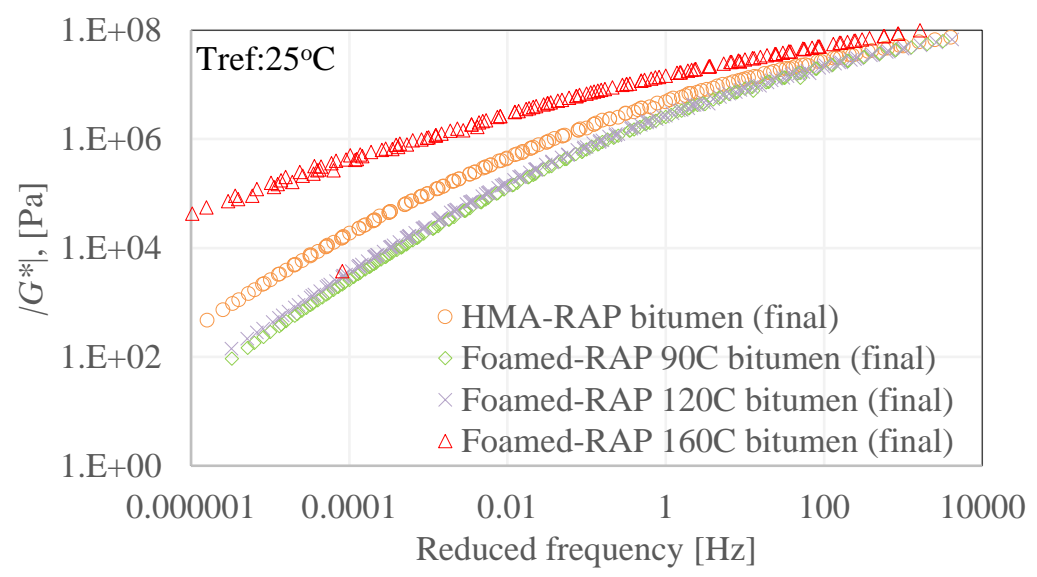

Figure 8 Master curves of $\left|G^{*}\right|$ for all recovered binders for a) initial condition, and b) after SATS conditioning

This figure shows that in all cases - before and after environmental conditioning - the bitumen recovered from the fine Foamed-RAP mixture manufactured at $160^{\circ} \mathrm{C}$ (Foamed-RAP 160C bitumen) exhibits the highest $\left|G^{*}\right|$ values, followed by the HMARAP bitumen, the Foamed-RAP 120C bitumen, and the Foamed-RAP 90C bitumen. This means that, different to what was observed from the corresponding FAM mixtures in Figure 6 , the ranking of the recovered binders in terms of their stiffness, did not change 
with the conditioning protocol. In order to compare the susceptibility to the environmental effects of the recovered binders to their corresponding FAM mixtures, $\left|G^{*}\right|$ ratios $\left(\left|G^{*}\right|_{\text {final }}\right.$ $/\left|G^{*}\right|_{\text {initial }}$ ) were calculated at a low frequency of $0.001 \mathrm{~Hz}$ (since higher changes in $\left|G^{*}\right|$ were observed at low frequencies), at the reference temperature of $25^{\circ} \mathrm{C}$ for the fine mixtures and their corresponding recovered binders for initial and final conditions. The results are presented in Figure 9. This figure includes the variability of the $\left|G^{*}\right|$ ratios for the FAM mixtures (i.e., vertical axis). The variability of the $\left|G^{*}\right|$ ratios for the recovered binders (i.e., horizontal axis) is not included, since only two replicates were tested for each recovered binder for the initial and final conditions.

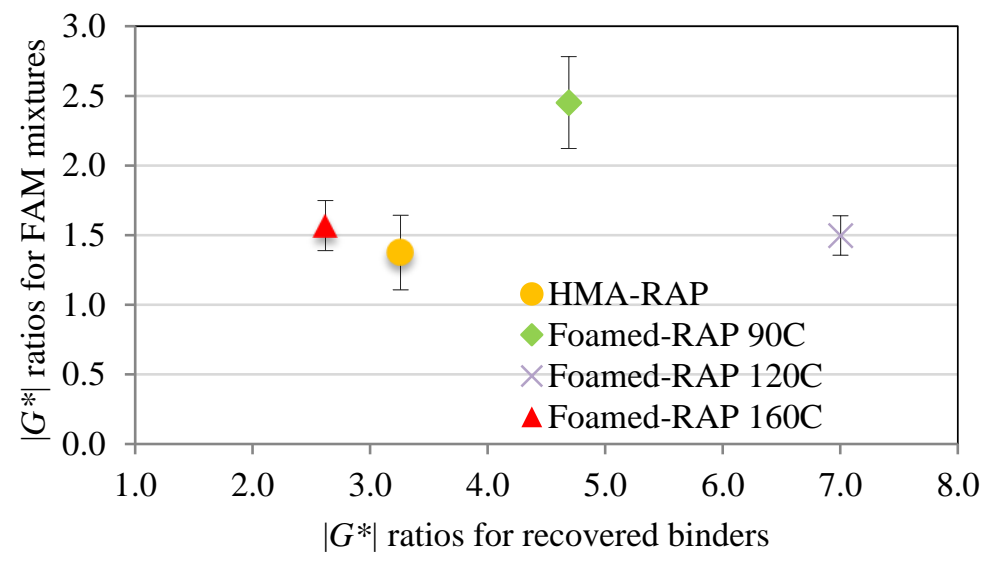

Figure $9\left|G^{*}\right|$ ratios for all FAM mixtures and their recovered binders

Figure 9 reveals that a change in the magnitude of the $\left|G^{*}\right|$ of the recovered binders does not lead to the same change in their corresponding FAM $\left|G^{*}\right|$, and that the ranking of $\left|G^{*}\right|$ ratios is not maintained (i.e., the recovered binder that exhibits the highest $\left|G^{*}\right|$ ratio does not correspond to the FAM that exhibits the highest $\left|G^{*}\right|$ ratio). Since the binder is responsible for the stiffening of the fine asphalt mixtures during oxidation, higher increase in the binder $\left|G^{*}\right|$ values are expected. However, the behaviour of the FAM materials is also affected by the aggregate skeleton and the internal void structure of the mixture, which can also explain the discrepancies between the FAM materials and their recovered binders.

The rheological data of the recovered binders prior and after conditioning was further used to calculate the Glover-Rowe $(G-R)$ parameter. The $G-R$ parameter captures the complex shear modulus $\left|G^{*}\right|$ and phase angle $(\delta)$ at a temperature-frequency combination of $15^{\circ} \mathrm{C}-0.005 \mathrm{rad} / \mathrm{s}$ (Equation 1) (Rowe, 2014), and it provides useful information about the long-term performance of the materials, particularly in their ability 
to resist cracking (King et al, 2012, Mensching et al, 2015; 2017). The results are generally plotted in the Black space (i.e., plot of $\delta$ vs. $\left.\left|G^{*}\right|\right)$. A higher $G-R$ value indicates increased brittleness and thus, higher susceptibility to cracking.

$$
G-R=\frac{\left|G^{*}\right|\left(\cos ^{2} \partial\right)}{\sin \partial}
$$

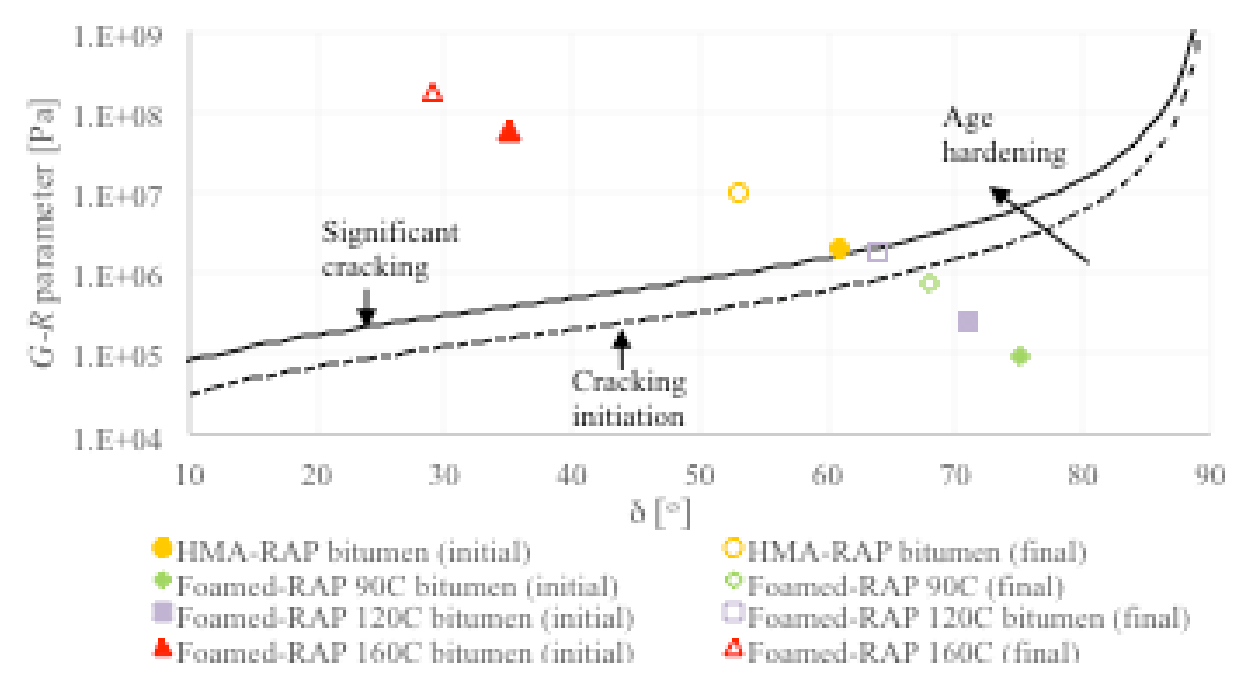

Figure $10 \mathrm{G}-\mathrm{R}$ parameter obtained for recovered binders before and after SATS test at $15^{\circ} \mathrm{C}$ and $0.005 \mathrm{rad} / \mathrm{sec}$

Figure 10 shows the $G-R$ and $\delta$ values, with the results of the recovered binders at the initial condition represented by filled markers, and those of the recovered binders after SATS conditioning represented by unfilled markers. The two curves shown in this figure (dotted and continuous curves) represent the $G-R$ parameter at values of $180 \mathrm{kPa}$ and 450 $\mathrm{kPa}$, respectively. These values correspond to the boundaries of expected crack initiation and significant cracking, respectively (Anderson et al. 2011). According to this analysis, the HMA-RAP and Foamed-RAP 160C recovered binders exceed the limit of cracking in both the initial and final conditions, which means that the fine mixtures containing these binders could have cracking-related issues in their long-term performance. It is also noticeable from this analysis that the rheological behaviour of the Foamed-RAP $120^{\circ} \mathrm{C}$ recovered bitumen after SATS conditioning is comparable to that of the HMA-RAP recovered binder at the initial condition - both binders are within the borderline of significant cracking. All other binders are still beneath them, suggesting that the experienced hardening effects will not compromise their cracking resistance.

These results indicate that there are two critical types of material responses after the conditioning protocol: 1) those where even though the $\left|G^{*}\right|$ ratio is high - indicating 
high increase in $\left|G^{*}\right|$ after the conditioning protocol, and thus high susceptibility to the environmental effects (e.g. Foamed-RAP 90 and Foamed-RAP 120C recovered binders, Figure 9) -, the magnitude of $\left|G^{*}\right|$ is within or does not exceed the cracking limits (Figure 10 ), and 2) those where the $\left|G^{*}\right|$ ratio is small, indicating that the material is not severely affected by the environmental effects (e.g. Foamed-RAP 160C and HMA-RAP recovered binders, Figure 9), but the actual magnitude of $\left|G^{*}\right|$ in both initial and final conditions exceeds the cracking limits, which could be a concern in terms of cracking susceptibility of the material. These results suggest that the change in the $\left|G^{*}\right|$ of the material between the initial and final condition, and the actual magnitude of the $\left|G^{*}\right|$ provide different but complementary information. Thus, both parameters must be considered to better assess the influence of environmental-related effects on the mechanical response of the materials and their long-term performance.

\section{Conclusions}

This study used the SATS conditioning protocol to evaluate combined environmental effects on the linear viscoelastic properties of a fine HMA-RAP mixture produced at $160^{\circ} \mathrm{C}$, and various fine Foamed-RAP mixtures produced at different mixing temperatures (i.e., 90, 120 and $160^{\circ} \mathrm{C}$ or half-warm, warm and hot applications, respectively). The impact of the environmental effects was also evaluated on the rheological properties of their recovered binders before and after being exposed to the SATS conditioning protocol. The main findings of this study are summarised below.

All fine mixtures exhibited a general increase in their $\left|G^{*}\right|$ values after the SATS conditioning protocol, with the Foamed-RAP and HMA-RAP fine mixtures produced at $160^{\circ} \mathrm{C}$ (with higher initial stiffness) exhibiting less increase in the magnitude of their $\left|G^{*}\right|$ values than the fine Foamed-RAP mixtures produced at 90 and $120^{\circ} \mathrm{C}$ (with less initial stiffness than the previous two). Thus, changes in production temperature of the fine mixtures had a significant effect on their environmental-related effects. This stiffening effect is mainly attributed to oxidation processes in the bitumen. Damage due to moisture (i.e., reduction in $\left|G^{*}\right|$ values due to adhesive failure between the fine aggregates bitumen interphase) was not observed, which was attributed mainly to the volumetric composition of the fine materials (i.e., high binder contents).

It was also observed that although the Foamed-RAP fine mixtures produced at 90 and $120^{\circ} \mathrm{C}$ were the most affected by the environmental conditioning (i.e., highest increase in their $\left|G^{*}\right|$ values), rheological analysis of their recovered binders show that this stiffening 
effect does not compromise their long-term performance in terms of cracking susceptibility. Conversely, the cracking susceptibility for the fine Foamed-RAP mixture produced at $160^{\circ} \mathrm{C}$ and the HMA-RAP fine mixture, although exhibited lower changes in $\left|G^{*}\right|$, in both initial and final conditions, could be a concern in their long-term performance. This means that a fair comparison between the environmental related effects of RAP-FBMs and their HMA-RAP counterparts should consider both the change of a certain property and the actual magnitude of those values, since both results are equally important.

In general, the combination of high RAP content (i.e., 50\%) with foaming techniques for half-warm and warm applications (i.e., mixing temperatures around 90 and $120^{\circ} \mathrm{C}$, respectively) might be a favourable sustainable approach for road infrastructure projects without compromising the long-term durability of the recycled mixtures. These results suggest that alternative approaches of the use of foamed bitumen by direct water injection with RAP material, such as half-warm and warm processes, which are different to that commonly used in the asphalt industry (i.e. cold recycling), could offer competent materials for asphalt mixtures to be used in surface courses.

Finally, it should be noted that the SATS test was originally developed for full asphalt mixtures with lower binder and higher air void content compared to those of the fine mixtures. Further research is needed to investigate a proper set of SATS conditions to reach higher saturation levels in fine mixtures with different bitumen and air void contents. In addition, the study should be complemented with testing and characterisation at the full mixture level.

\section{Acknowledgements}

The first author would like to thank Colciencias for the funding provided through the program No. 617-2013 for doctoral studies abroad, and the partial funding provided by the Nottingham Transportation Engineering Centre (NTEC).

\section{ORCID}

Diana B. Sánchez http://orcid.org/0000-0001-7680-5444

Silvia Caro http://orcid.org/0000-0003-2726-3575

\section{References}

Abbas, A., Nazzal, M., Kaya, S., Akinbowale, S., Subedi, B., Shahnewaz Arefin, M., Abu, L. (2016). Effect of Aging on Foamed Warm Mix Asphalt Produced by Water Injection. Journal of Materials in 
Civil Engineering, 28 (11), 04016128-1-11.

Airey, G. D. (2002). Use of black diagrams to identify inconsistencies in rheological data use of black diagrams to identify inconsistencies in rheological data. Road Materials and Pavement Design, 3 (4), 403-424.

Airey, G.D. (2003) State of the art report on ageing test methods for bituminous pavement materials, International Journal of Pavement Engineering. 4 (3) 165-176.

Airey, G. D., Choi, Y. K., Collop, A.C., Moore, A. J. V., \& Elliott, R.C. (2005). Combined laboratory ageing / moisture sensitivity assessment of high modulus base asphalt mixtures. Journal of the Association of Asphalt Paving Technologists, 74, 307-346.

Al-Qadi I., Elseifi, M., Carpenter, S. (2007) Reclaimed asphalt pavement - A literature review. Illinois Center for Transportation (ICT), Springfield, Illinois. Report No. FHWA-ICT-07-001

Anderson, M., King, G., Hanson, D., and Blankenship, P. (2011). Evaluation of the relationship between asphalt binder properties and non-load related cracking. Journal of the Association of Asphalt Paving Technologists, 80, 615-664.

BS 2000-49:2007. Bitumen and bituminous binders - determination of needle penetration. British Standards Institution (BSI) Publication.

BS 2000-505:2010. Bitumen and bituminous binders - determination of dynamic viscosity of bituminous binder using a rotating spindle apparatus. British Standards Institution (BSI) Publication.

BS 2000-58:2007. Bitumen and bituminous binders - determination of the softening point - ring and Ball method. British Standards Institution (BSI) Publication.

BS 2000-80:2007. Bitumen and bituminous binders - determination of the Fraass breaking point. British Standards Institution (BSI) Publication.

BS 4987-1:2005. Coated macadam (asphalt concrete) for roads and other paved areas - Part 1: Specification for constituent materials and for mixtures. British Standards Institution (BSI) Publication.

BS EN 12697-31:2007. Bituminous mixtures - test methods for hot mix asphalt - Part 31: Specimen preparation by gyratory compactor. British Standards Institution (BSI) Publication.

BS EN 12697-4:2005. Bituminous mixtures - test methods for hot mix asphalt - Part 4: Bitumen recovery: Fractionating column. British Standards Institution (BSI) Publication.

Bonaquist, R. (2005). Laboratory evaluation of hot mix asphalt (HMA) mixtures containing recycled or waste product materials using performance testing (FHWA-PA-2005-006-98-32(19)). Pennsylvania: Department of Transportation, Office of Planning and Research.

Caro, S., Beltrán, D. P., Alvarez, A. E., \& Estakhri, C. (2012). Analysis of moisture damage susceptibility of warm mix asphalt (WMA) mixtures based on dynamic mechanical analyzer (DMA) testing and a fracture mechanics model. Construction and Building Materials, 35, 460-467.

Caro, S., Masad, E., Airey, G., Bhasin, A., \& Little, D. (2008). Probabilistic analysis of fracture in asphalt mixtures caused by moisture damage. Transportation Research Record: Journal of the Transportation Research Board, 2057, 28-36.

Caro, S., Masad, E., Bhasin, A. \& Little, D.N. (2008). Moisture susceptibility of asphalt mixtures, Part 1: mechanisms. International Journal of Pavement Engineering, 9, 81-98.

Caro, S., Sánchez, D. B., \& Caicedo, B. (2015). Methodology to characterise non-standard asphalt materials using DMA testing: Application to natural asphalt mixtures. International Journal of Pavement Engineering, 16, 1-10. 
Collop, A. C., Choi, Y., Airey, G. D. \& Elliot, R. C. (2004). Development of the Saturation Ageing Tensile Stiffness (SATS) Test. Proceedings of the Institution of Civil Engineers (ICE), 157 (3), 163-171.

Corrigan, M. (2008). Warm mix asphalt technologies and research. US. Department of Transportation. Federal Highway Administration-FHWA. Washington D.C.

D’Angelo, J., Harm, E., Bartoszek, J., Baumgardner, G., Corrigan, M., Cowsert, J., Harman, T., Jamshidi, M., Jones, W., Newcomb, D., Prowell, B., Sines, R. \& Yeaton, B. (2007). Warm-mix asphalt: European practice. Report No. FHWA-PL-08-007. Federal Highway Administration. Department of Transportation, Washington, DC.

EN 13108-8:2005. Bituminous mixtures - material specifications - Part 8: Reclaimed asphalt.

Figueroa Infante, A.S. 2015. Investigación sobre el efecto del agua en el asfalto y su impacto en la mezcla asfáltica. PhD Thesis. Pontificia Universidad Javeriana. Bogotá, Colombia.

Grenfell, J., Ahmad, N., Airey G. D. \& Elliott, R. (2012). Optimising the moisture durability SATS conditioning parameters for universal asphalt mixture application. International Journal of Pavement Engineering, 13, 433-450.

Gudipudi, P., \& Underwood, B. S. (2015). Testing and modeling of fine aggregate matrix and its relationship to asphalt concrete mix. Transportation Research Record: Journal of the Transportation Research Board, 2507, 120-127.

Jacques, C., Daniel, J. S., Bennert, T., Reinke, G., Norouzi, A., Ericson, C., Mogawer, W. \& Kim, Y.R. (2016). Effect of silo storage time on the characteristics of virgin and RAP asphalt mixtures.

Transportation Research Record: Journal of the Transportation Research Board, No. 2573. Washington, D.C, $76-85$

Karlsson, R. \& Isacsson, U. (2006). Material-Related Aspects of Asphalt Recycling-State-of-the-Art. Journal of Materials in Civil Engineering (ASCE), 18(1), 81-92.

Kavussi, A., Motevalizadeha, M., Karimi, A., \& Rahimizadeh, A. (2017). Evaluating the Moisture Resistance of Foam Warm Mix Asphalt Using Image Processing Method. Computational Research Progress in Applied Science \& Engineering (CRPASE), 3, 1-7, Special Issue: 1st National Conference on Highway \& Transportation Engineering.

Kennedy, T.W., Roberts, F.L., \& Lee, K.W. (1983). Evaluation of moisture effects on asphalt concrete mixtures. Transportation Research Record, 911, 134-143.

Kim, Y., Zhang, J., \& Ban, H. (2012) Moisture damage characterization of warm-mix asphalt mixtures based on laboratory-field evaluation. Construction and Building Materials, 31, 204-211.

King, G., Anderson, M., Hanson, D., \& Blankenship, P. (2012) Using black space diagrams to predict age-induced cracking, in: 7th RILEM International Conference on Cracking in Pavements, Springer, 453463.

Klug, A. B., Ng, A. K. Y., \& Faxina, A. L. (2018). Evaluation of the fatigue performance of fine aggregate matrices prepared with reclaimed asphalt pavements and shale oil residue. Transportation research board annual meeting, Washington, DC.

Kusam, A., Malladi, H., Tayebali, A.A, \& Khosla, N.P. (2017). Laboratory evaluation of workability and moisture susceptibility of warm-mix asphalt mixtures containing recycled asphalt pavements. Journal of Materials in Civil Engineering, 29 (5).

López-Montero, T., \& Miró, R. (2016). Differences in cracking resistance of asphalt mixtures due to ageing and moisture damage. Construction and Building Materials 112, 299-306.

Ma, T., Huang, X., Mahmoud, E., Garibaldy, E. (2011). Effect of moisture on the aging behavior of asphalt binder. International Journal of Minerals, Metallurgy and Materials, 18 (4), 460-466. 
Mallick, R. B., \& Hendrix, G. (2004). Use of foamed asphalt in recycling incinerator ash for construction of stabilized base course. Resources, Conservation and Recycling, 42(3), 239-248.

Masad, E., Castelo Branco, V. T. F., Little, D. N., \& Lytton, R. (2008). A unified method for the analysis of controlled-strain and controlled-stress fatigue testing. International Journal of Pavement Engineering, 9, 233-246.

Masad, E., Zollinger, C., Bulut, R., Little, D., \& Lytton, R. (2006). Characterization of HMA moisture damage using surface energy and fracture properties. Journal of the Association of Asphalt Paving Technologists (AAPT), 75, 713-754.

Mensching, D. J., Rowe, G. M., Daniel, J. S., \& Bennert, T. (2015). Exploring low-temperature performance in Black Space. Road Materials and Pavement Design, 16(sup2), 230-253.

Miller, J.S. \& Bellinger, W.Y. (2003). Distress identification manual for the long-term pavement performance program. Report No. FHWA-RD-03-031. McLean, VA: FHWA, Office of Infrastructure Research and Development.

Mogawer, W., Bennert, T., Daniel, J. S., Bonaquist, R., Austerman, A., \& Booshehrian, A. (2012). Performance characteristics of plant produced high RAP mixtures. Road Materials and Pavement Design, 13(sup1), 183-208.

Namutebi, M., Birgisson, B., \& Bagampadde, U. (2011). Foaming Effects on Binder Chemistry and Aggregate Coatability using Foamed Bitumen. Road Materials and Pavement Design, 12(4), 821-847.

Oke, O. O. (2011). A study on the development of guidelines for the production of bitumen emulsion stabilised RAPs for roads in the tropics. PhD Thesis. The University of Nottingham. Nottingham, UK.

Oyebisi, S (2014). Influence of aging on foamed warm mix asphalt. MSc Thesis. The University of Akron. Akron, $\mathrm{OH}$.

Raab, C., Camargo, I., \& Partl, M. N. (2017). Ageing and performance of warm mix asphalt pavements. Journal of Traffic and Transportation Engineering (English Edition), 4(4), 388-394.

Rowe, G., Baumgardner, G., \& Sharrock, M. (2009). Functional Forms for Master Curve Analysis of Bituminous Materials. Proceedings of 7th International RILEM 229 Symposium ATCBM09 on Advanced Testing and Characterization of Bituminous Materials, 1, pp. 81-91.

Sánchez, D. B., Grenfell, J., Airey, G., \& Caro, S. (2017). Evaluation of the degradation of fine asphaltaggregate mixtures containing high reclaimed asphalt pavement contents. Road Materials and Pavement Design, 18(sup2), 91-107.

Sánchez, D. B., Airey, G., Caro, S., \& Grenfell, J. (2019). Effect of foaming technique and mixing temperature on the rheological characteristics of fine RAP-foamed bitumen mixtures. Road Materials and Pavement Design, 1-17.

Shah, A., McDaniel, R.S., Huber, G.A., \& Gallivan, V.L. (2007). Investigation of properties of plant produced reclaimed asphalt pavement mixtures. Transportation Research Record: Journal of the Transportation Research Board, 1998, 103-111.

Tarbox, S., \& Daniel, J. S. (2012). Effects of Long-Term Oven Aging on Reclaimed Asphalt Pavement Mixtures. Transportation Research Record: Journal of the Transportation Research Board, 2294(1), 1-15.

Tong, Y., Luo, R., \& Lytton, R. L. (2013). Modeling water vapor diffusion in pavement and its influence on fatigue crack growth of fine aggregate mixture. Transportation Research Record: Journal of the Transportation Research Board, 2373, 71-80.

Tong, Y., Luo, R., \& Lytton, R. L. (2015). Moisture and aging damage evaluation of asphalt mixtures using the repeated direct tensional test method. International Journal of Pavement Engineering, 16, 397410. 
Tran, N.H., Taylor, A. \& Willis, R., (2012). Effect of Rejuvenator on Performance Properties of HMA Mixtures with High RAP and RAS Contents. NCAT Report 12-05, National Center for Asphalt Technology, Aurburn University, Auburn, Alabama.

Underwood,B.S.\& Y.R.Kim. (2013). MicrostructuralInvestigationofAsphaltConcretefor Performing Multiscale Experimental Studies. International Journal of Pavement Engineering, Vol. 14, No. 5, 498-

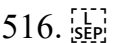

Yin, F., Arámbula-Mercado, E., Epps Martin, A., Newcomb, D., \& Tran, N. (2017). Long-term ageing of asphalt mixtures. Road Materials and Pavement Design, 18(sup1), 2-27. 